\title{
Polymer-based surface plasmon resonance biochip: construction and experimental aspects
}

Cleumar da Silva Moreira*, Leiva Casemiro Oliveira, Robert Fischer, Eliton Souto Medeiros, Antonio Marcus Nogueira Lima, Helmut Neff

\begin{abstract}
Introduction: Surface plasmon resonance biosensors are high sensitive analytical instruments that normally employ glass materials at the optical substrate layer. However, the use of polymer-based substrates is increasing in the last years due to favorable features, like: disposability, ease to construction and low-cost design. Review: Recently, a polymer-based SPR biochip was proposed by using monochromatic and polychromatic input sources. Its construction and experimental considerations are detailed here. Experimental considerations and results, aspects from performance characteristics (resonance parameters, sensitivity and full width at half maximum - FWHM - calculations) are presented for hydrophilic and hydrophobic solutions. It is included also a brief description of the state of the art of polymer-based SPR biosensors.
\end{abstract}

Keywords: Polymer-based SPR, Biochip, Biosensor, Surface plasmon resonance.

\section{Introduction}

Since 1983, when the first surface plasmon resonance (SPR) biosensor was proposed (Liedberg et al., 1983), the use of the SPR phenomenon for biosensing is widely reaching several applications which can be referred by recent reviews (Arima et al., 2011; Hoa et al., 2007; Rusnati and Presta, 2015; Šípová and Homola, 2013; Situ et al., 2010; Thillaivinayagalingam et al., 2010). This occurs due to the advantages of the SPR biosensors, like: label-free method, ease of miniaturization, fast response (minutes to some hours, when compared to conventional bioanalytical methods, like ELISA) and high sensitivity and specificity (Moreira, 2010; Oliveira, 2011). Actually more than ten commercial SPR instruments are available on the market (Mukhopadhyay, 2005; Schasfoort and Tudos, 2008).

For SPR phenomenon occurrence is necessary a dielectric-metal interface, where free electrons behaving as longitudinal oscillations can be excited impinging light in the metal side, according certain specific conditions. One of these conditions is the use of an optical substrate to couple the incoming light to these oscillations (Otto, 1968; Raether, 1988). These optical substrates are normally made of a glass or polymeric materials in different arrangements, like waveguides, optical fibers, prisms, etc. (Homola, 2006; Maier, 2007).

Here, a SPR polymeric biochip is presented as an alternative for diagnostic and point of care applications. Technical considerations on the design, construction, experimental results and comparison with a commercial device will be also presented.

\section{Theoretical aspects}

In 1968, Otto suggested that a reflectance reduction that has been observed earlier in 1902, for a metal-dielectric interface, is provoked by the interaction between the incident photons and longitudinal oscillations on the surface that were named surface plasmons or SP (Otto, 1968). He proposed a structure for light-based controlled excitation of surface plasmon that was composed by the following layers: an optical prism for light coupling; a dielectric (air); and a metallic film. The Otto's structure has not become popular due to the strong dependence of the SP coupling on the thickness of the dielectric. On the other hand, this configuration is useful to the investigation of phonons polarization on a simple crystal surface and to the thickness measurement of thin films (Raether, 1988).

Also in 1968, Kretschmann and Raether adapted Otto's configuration and proposed a modified configuration called Attenuated Total Reflection structure or simply ATR structure. It used a glass optical prism to couple the incoming light beams into the metal-dielectric interface. Although other surface plasmons excitation techniques can be used, like an end-fire coupling or by using a diffraction grating, the prism-based coupling is simpler and needs less tuning. Based on the ATR 
structure proposal, SPR technology primarily was used for thin film characterization.

Only in 1983, a Swedish group proposed the first SPR biosensor applied to gas detection. They used a classical glass prism and a monochromatic light source to excite SPs in a 50-nm gold thin film (Carvalho et al., 2006; Liedberg et al., 1983). Since this first SPR biosensor, many commercial and non-commercial SPR devices have been proposed for several application fields, such as in pharmaceutical and medical industries and environmental monitoring (Hu et al., 2009; Kumbhat et al., 2010; Myszka and Rich, 2000).

Attempts to integrate SPR biosensor apparatus, in order to reduce size and cost, have been proposed, like the use of: polymer-based prisms, using packaged (Chinowsky et al., 2003; Chinowsky et al., 2007; Elkind et al., 1999) or non-packaged arrangements (Loureiro et al., 2009; Loureiro et al., 2011; Moreira et al., 2009 and Thirstrup et al., 2004), conventional (Cennamo et al. 2013) and microstructured (Dash and Jha, 2014) optical fibers, silicon-based devices (Ghosh and Ray, 2015 and Patskovsky et al., 2003) and nanoparticles-based devices (Atar et al., 2015; Rivero et al., 2012; Sener et al., 2011 and Willets and Van Duyne, 2007). For the nanoparticles-based devices the associated physics is different than SPR physics and the phenomenon is known as Localized SPR or LSPR.

\section{SPR theory}

Optical properties for metals can be explained by Drude theory, which states that a free electron gas is moving through a fixed array of positive ions defined by the following dielectric function (Maier, 2007):

$$
\varepsilon(\omega)=1-\frac{\omega_{p}^{2}}{\omega^{2}+i \cdot \gamma \cdot \omega}
$$

where

$\omega_{p}^{2}=\frac{n e^{2}}{\varepsilon_{0}}$

is the plasmon frequency of free electron gas, $\gamma$ is the damping rate, $n$ is the number of electrons, $e$ is electron charge and $\varepsilon_{0}$ is the vacuum permittivity.

The free electron oscillation occurs only for frequencies higher or equal to $\omega_{p}$ (Maier, 2007), where the quanta of the oscillations are called plasmon or volume plasmon. When these oscillations are on the metal surface, they are named surface plasmon polaritons (SPPs) or simply surface plasmons (SP). The SPs are sensitive to input radiation and normally the injection of electrons (application of an electrical current) or light beam injection (photons) are preferred to excite them (Raether, 1988). For light excitation, that is a simpler method, the application range varies from near infrared to visible light (Maier, 2007).

When a p-polarized light beam, with wave vector $\left(k_{x}\right)$ parallel to the metal-dielectric interface, impinges on the interface, the incoming photons are transmitted and interact with surface plasmons $\left(k_{s p}\right)$. An evanescent electric field $\left(E_{e v}\right)$ arises from photons transmission with a small penetration depth and a maximum value that is found on the surface. Therefore, any modifications on the electro-optical properties of the substances close to the interface are easily detected. This leads to SPR technology to be very attractive for biosensing applications (Homola, 2006; Schasfoort and Tudos, 2008).

To describe the interaction of photons with surface plasmons, an approximation is normally employed, where the dissipative imaginary components of the complex dielectric functions $\left(\varepsilon=\varepsilon^{\prime}+\mathrm{i} \varepsilon^{\prime}\right)$ involved, are neglected. The optical materials parameters are steady functions of wavelength and temperature (Moreira et al., 2008). The general form of the resonance condition reads as:

$$
\frac{2 \pi}{\lambda} \sqrt{\varepsilon_{2}} \sin \left(\theta_{\mathrm{Res}}\right) \cong \frac{2 \pi}{\lambda} \sqrt{\frac{\varepsilon_{\mathrm{mr}} \varepsilon_{\mathrm{S}}}{\varepsilon_{\mathrm{mr}}+\varepsilon_{\mathrm{S}}}}
$$

Both sides of Equation 3 correspond to, and describe matching of the complex photon $k_{p h}$ and plasmon $k_{p l}$ wave vectors. $\lambda$ denotes the wavelength of the TEM (p)-polarized light, $\varepsilon_{2}(\lambda), \varepsilon_{m r}(\lambda)$ and $\varepsilon_{S}(\lambda)$ are the individual wavelength dependent complex dielectric functions of an optically transparent substrate, the semi-transparent gold metal film, and an adjacent aqueous analyte solution, respectively. The general form would be required, if optically highly absorbing analyte solutions, as blood and other liquids, would be involved. Analysis of the aforementioned general form of the resonance condition, employing software assisted complex symbolic calculus, indicated that it principally should be possible to solve for $\theta_{\text {Res }}(\lambda)$. Among other problems, the general notation of the resonance condition requires introduction of a complex resonance angle, and leads to exceedingly long and difficult treatable mathematical expressions for the below listed physical quantities (Moreira et al., 2008).

Solving (3) for $n_{S}$, and use of only the real part of the dielectric functions, with $n=\sqrt{\varepsilon}$, provides a simplified expression for the real part of the refractive index $n_{S}$ :

$n_{S}=\left[\frac{\varepsilon_{m r}\left(n_{2} \sin \theta_{r e s}\right)^{2}}{\varepsilon_{m r}-\left(n_{2} \sin \theta_{r e s}\right)^{2}}\right]^{1 / 2}$ 
where $\theta_{\text {res }}$ represents the aforementioned real resonance angle, as measured by the SPR sensor, associated with the minimum in the light intensity reflected from the metal film. Variations of the refractive index of the analyte $n_{\mathrm{S}}$ are taken into consideration by $n_{S}=\alpha * n_{3}(0.95<\alpha<1.05)$, where $\mathrm{n}_{3}$ denotes the refractive index of water (1.332) at ambient T (298 K) and wavelength of $670 \mathrm{~nm}$.

Moreover, it can be shown that neither intersection occurs between the input light and surface plasmon wave vectors (Moreira, 2010). For this condition, it is not possible to directly excite the surface plasmon and a light-coupling medium is required. By using this light-coupling layer, the input wave vector magnitude increases and an intersection takes place. This coupling layer is normally made of a glass or polymeric materials in different arrangements, like waveguides, optical fibers, prisms, etc.

\section{SPR biosensor}

In a typical SPR biosensor, the optically sensed quantity $n_{S}$ cannot be treated as a homogeneous semi-infinite phase, but must be considered as a non-homogeneous distributed medium, with optical properties exhibiting distinct spatial variations.
Such modulations in the optical boundary conditions occur in presence of an addition bio / chemical film, attached to a linker or functionalized molecular surface film at molecular thickness range. A four layers model is considered appropriate to describe the optical arrangement: A semi-infinite transparent dielectric substrate; a semi-transparent metal film; a bio-/chemical film; and a neighboring aqueous semi-infinite medium

The bio-/ chemical film deposited onto the thin metal-film would grow out, and immobilize onto the gold metal surface from an admitted aqueous solution, containing the bio-/chemical substance as a solvent. Fresnel equations, treating an optical multi-layer system by means of a matrix representation is generally employed for analysis, described in detail elsewhere (Neff et al., 2005). The physical information of interest is film thickness $d_{3}$ and/or the associated refractive index.

For SPR biosensor, the instrumental design comprises four key components (Figure 1a):

- A set of appropriately designed and arranged optical elements for radiation generation, beam forming/ guiding and angle or spectrally resolved optical detection;

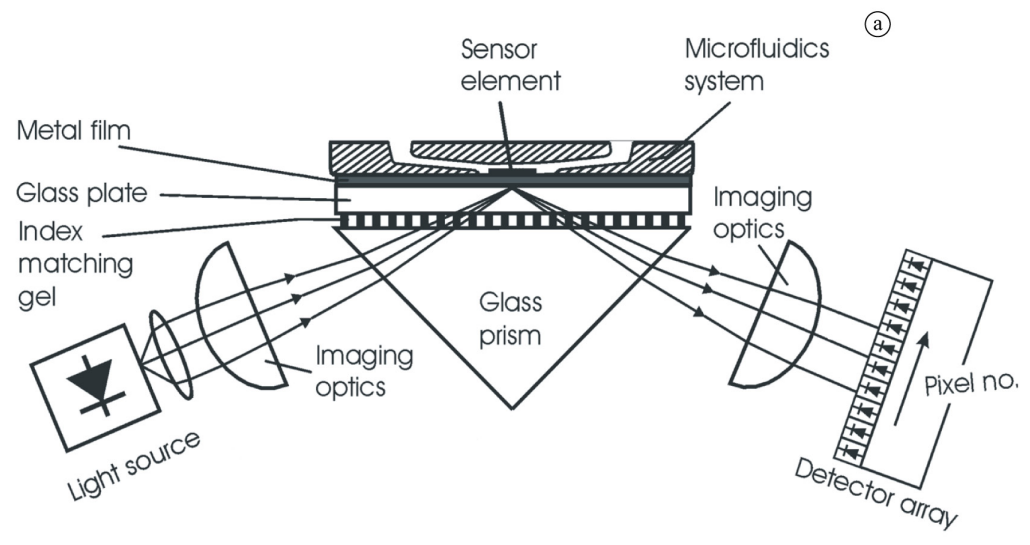

(b)
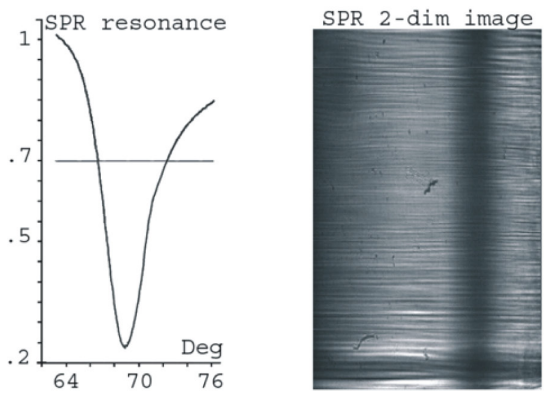

Figure 1. (a) Schematic instrumental set-up of the Kretschmann configuration, employed for angularly resolved surface plasmon resonance sensing in Figure 1a; (b) the resonant SPR absorption line and derived profile of the so-called SPR dip as a function of angle. 
- A sensing surface / device for plasmon excitation, comprising total optical reflection at a thin semi-transparent metal film. This leaves a sharply defined and optically absorption line, illustrated in Figure 1b. Its spectral or angular position changes, as the optical boundary conditions vary;

- A set of hydrodynamic elements, comprising a flow cell connected to the sensing surface, tubes and pump, to deliver the liquid medium (the analyte) under investigation to the sensing surface;

- Computer assisted data processing and display (not shown).

A SPR biosensor can be optically operated by monochromatic or polychromatic input light sources, where the wavelength interrogation and angular interrogation modes are commonly proposed at commercial and non-commercial devices (Schasfoort and Tudos, 2008). The later uses a polychromatic source (normally a halogen source or a phosphor- or RGB-based white light diodes) and a spectrometer as the detector, varying the light wavelength and keeping constant the incidence angle (Homola et al., 2005; Wang et al., 2004). The former uses a monochromatic source and a camera as the detector, varying the incidence angle and keeping constant the light wavelength (Guo et al., 2008).

\section{Polymer-based SPR Devices}

Since the first SPR biochip (that is formed by the optical substrate and the metal thin film) in 1983 (Liedberg et al, 1983), glass substrates have been largely used as the substrate material (Le Person et al., 2008; Sexton et al., 2008, Yu et al., 2014). Nevertheless, use of polymers as substrates for SPR devices has grown in the last few years, motivated mainly by their low-cost design and ease of construction. These biochips can be typically produced by injection molding, which is simple but involves the use of a mold, whose price is relatively high, especially if few prisms need to be produced. The use of optical fiber substrates avoids the injection molding technique, but the manufacturing process is more complicated and expensive (Cennamo and Zeni, 2014; Sharma et al., 2007).

Some commercial polymeric biochips have been proposed in the last decades. Texas Instruments launched the first SPR polymeric biochip that was called SPREETA (Chinowsky et al., 2003; Elkind et al., 1999). All electro-optical elements were packaged in the same polymer enclosure, which provided safety and appropriate conditions for SPR phenomenon to occur on the nanometallic layer. Although a SPREETA biochip has a minor cost in comparison with other commercial biochips, like BIACORE (manufactured by GE Healthcare) that is the most propelled in health institutions and universities, its use is still restricted especially due especially to the high temperature dependence (Moreira et al., 2008; Moreira, 2010).

Another commercial SPR sensor chip proposed in 2004 (Neff et al., 2005; Thirstrup et al., 2004), called VIRChip uses a flat disposable optical chip, equipped with two integrated focusing / defocusing diffractive grating elements (DOCE's) for internal beam forming and guiding to and from the sensing surface. The optical chip is made from TOPAS polymer material, producible in a high volume injection molding process, thus accounting for very low chip cost. The set-up leads to drastic reduction in instrumental volume, simplified optical design and practically maintenance free operation (Thirstrup et al., 2004). VIRChip has been applied only for AIM applications. For its manufacturing, a high upfront cost is required for the initial mold (Loureiro et al., 2009, 2011; Moreira, 2010; Oliveira, 2011; Souza, 2006).

Another example of a polymer-based SPR biosensor has been proposed using the same polymer of VIR biochip (Moreira, 2010). This device constitutes the first polymer-based SPR biochip manufactured in Brazil and it was named PPBIO. It has a simple structure and can be applied for both AIM and WIM, and requires relatively low design and manufacturing costs (Moreira et al., 2009; Moreira, 2010):

Recently a new SPR biochip for AIM and WIM measurements was proposed, based on the Kretschmann model (Kasztelanic, 2012). This improvement in functionality is considered the main contribution of the work. Also, due to the setup configuration, temperature effects can also be compensated.

Some SPR biosensor configurations using polymer optical fiber as the substrate have been also proposed (Dash and Jha, 2014; Cennamo and Zeni, 2014). One way to construct a plastic optical fiber SPR biosensor, which is normally used, is removing the cladding material of a certain region of the substrate and replacing it with a metallic one. This cladding removal leads to the evanescent field being propagated with higher intensity along the dielectric, which also increases the sensitivity (Cennamo and Zeni, 2014). Different proposals have been presented to remove the cladding: grinding and polishing; use of laminated; laser injection, acetone and other chemical elements or solutions (Kuang et al., 2002; Vasile et al., 2013). Recently, an acetone: alcohol mixture $(1: 1)$ have been proposed with optimum results, where after an exposition time of $8 \mathrm{~min}$ the cladding material was easily removed without damaging the core and a gold thin film was sputtered on the core (Santiago et al, 2015). 
Scanning Electronic Microscope (SEM) images of the core after removal of the cladding material show a fairly smooth surface with neither bubbles nor mechanical stresses.

SPR optical fibers biosensors offer good advantages, like miniaturization and possibility of being used in harsh environments, while maintaining good reliability and performance. One disadvantage of these configurations is the biochip cost when compared to those manufactured by injection molding technique. On the other hand, these configurations possess a small size and therefore are adequate for portable applications.

\section{Case Study}

PPBIO SPR biochip will be detailed here as a case study, where its design and construction considerations and experimental results will be presented. PPBIO has promising characteristics for a portable analytical device proposal: simple geometry, low cost and disposability.

\section{Material and methods}

The SPR biochips to be fabricated are composed by two phases: one is the polymeric prism made by injection molding process and the other is the thin film metallic deposition. At the manufacturing of the polymeric prisms (a sketch of the prism and its dimensions are depicted in Figure 2a), a stainless steel mold with 4 cavities, according Figure $2 b$, have been machined with a standard CNC machine. At the preform, the cavities, electrodes and the wells have been machined. Also for the platen, the machining of preform housing, the cooling holes, the centering ring housing, the injection nozzle, support plate and extractor plates holes for pin pullers have been made. Other items that have been machined were the sprue bushing, extractor pin branch for lathe and mill and the nozzle inlet and outlet water.
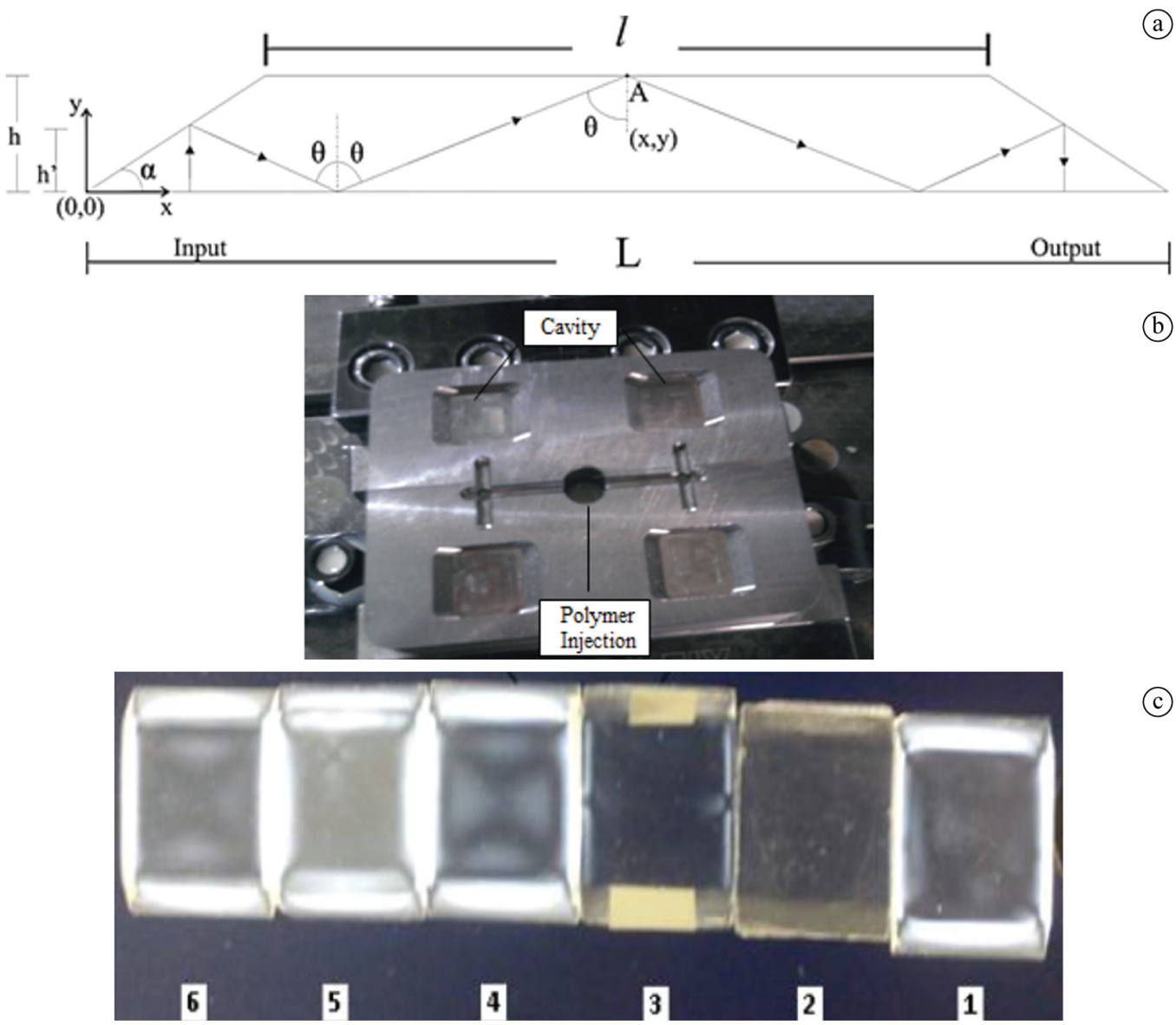

(b)

(a)

Figure 2. (a) Prism schematic diagram, with dimensions and geometric localization; (b) picture of the four cavities mold; and (c) picture of six different PPBIO showing the birefringence effects. 


\section{Design and construction aspects}

The prism is shown in Figure 2c through pictures, showing birefringence effects, with and without gold deposition. A $100-\mathrm{nm}$ silver thin film covers the inclined surfaces and facilitates the internal reflection.

The trapezoidal prism uses a polymer commercially named TOPAS COC. This polymer is amorphous and it has a high glass transition temperature (higher than $200^{\circ} \mathrm{C}$ ), a high mechanical resistance and transparence. Also, it has a low birefringence, a hydrophobic characteristic, a good thermal stability, chemical resistance and a low density

The manufacturing of the prism biochip embodies all the relevant choices for the providing the SPR effect excitation. The procedure is divided into two stages: first one aiming at the prism geometry fabrication, i.e., the choice for the substrate optical of the multilayer structure; and the second one dealing with the metal deposition on the prism surface, i.e., the choice for the thin metallic layer. The distance travelled by the light after being reflected at the left side mirror until it reaches middle point where the top surface determines the physical dimensions of the prism biochip and the incidence angle at the metallic layer.

In the design of the prism biochip it is assumed that the incidence angle of the light is $68^{\circ}$. In this case the resonance condition occurs at a resonance wavelength $\lambda_{R}=670 \mathrm{~nm}$, only when deionized water flows over the metallic layer. This is the Operating Point (OP) of the PPBIO. When gold is replaced by silver in the AIM configuration, $\theta_{R}$ changes to $66^{\circ}$. When BK7 is used instead of TOPAS $\theta_{R}$ changes to $69.5^{\circ}$ and $67^{\circ}$ for gold and silver, respectively.

At the condition described in the previous paragraph, the PPBIO dimensions are (Figure 2a): height (h) equal to $3 \mathrm{~mm}$, top length (1) is $17.84 \mathrm{~mm}$, bottom length, $\mathrm{L}=26.78 \mathrm{~mm}$ and inclination side angle, $\alpha=34^{\circ}$. To obtain this precise geometry, an injection mold (Figure $2 b$ ) was designed and used. The four cavities comprise exactly the original geometry, i.e., when gold thin metal layer are deposited on PPBIO.

To maintain the incident light polarization state and the incident light lenses focalization as well to improve the prism performance by emphasizing the resonance dip and reducing transmission losses, annealing and polishing are required after the PPBIO is extracted from the mold. Figure $2 \mathrm{c}$ shows the sample shed by a p-polarized light. Prism without annealing $(1,4,5,6)$ changes the polarization and then degrades the SPR excitation. Without polish $(1,2,5,6)$, the amount of light attenuated along the path affect the depth of SPR curve dip.
The PPBIO was designed to provide a low-cost instrumentation due to its trapezoidal geometric characteristics, allowing light beam have a perpendicular incidence in the bottom face of the PPBIO. Changes in the manufacturing aspects of PPBIO, in the metal layer, have produced changes on the operating points of the PPBIO and consequently changes in calibration process are present.

Also, due to the intrinsic construction (injection molding) aspects, irregularities and high levels of stress have been noted. To minimize this effect, the biochip prisms have heated until relaxation stress temperature, about $250^{\circ} \mathrm{C}$ for 24 hours, and controllably cooled to room temperature, in a process called annealing. The biochips without proper annealing do not exhibit the typical SPR resonance dip (Oliveira et al., 2013; Oliveira et al., 2014a). Also, the prism polishing improves the device performance by emphasizing the resonance dip and reducing transmission losses. Before polishing the absorbance at $655 \mathrm{~nm}$ was about to 0.126 , which represents a reduction of $18.19 \%$ in light transmitted through the biochip. For the same wavelength, after polishing the absorbance has been reduced to 0.035 , reducing the transmission losses to $8.5 \%$.

Other design possibility to $\mathrm{PPBIO}$ has been recently proposed (Oliveira et al., 2014b) and involves the use of bimetallic thin layers instead of using a gold monolayer. This new arrangement maintains the prism geometry unchanged and also permits easily adjust or calibrate OP by using a parameter that is expressed by the reason between the detectivities on angular and wavelength modes. According simulation results, the sensitivity was enhanced using the combination $\mathrm{Au} / \mathrm{Cu}$ for $\mathrm{AIM}$ and $\mathrm{Au} / \mathrm{Ag}$ for WIM.

\section{Experimental aspects}

The experiments (test cycle) attempt to check some parameters (sensitivity, FWHM - Full Width at Half-Maximum, resonant wavelength and angle) that are important to characterize the occurred phenomenon or the associated performance of the biosensor.

Sensitivity is a figure of merit that is defined at AIM and WIM cases as, respectively

$$
\begin{gathered}
S_{n_{3}}^{\theta_{R}}=\frac{\partial \theta_{R}}{\partial n_{3}} \\
S_{n_{3}}^{\lambda_{R}}=\frac{\partial \lambda_{R}}{\partial n_{3}}
\end{gathered}
$$

Follows the obtained results, by considering two procedures: the first used hydrophilic (reversible bindings) and hydrophobic (non-reversible bindings) 
analytes for the calculation of the SPR curve parameters $\left(\lambda_{\text {RES }}, \theta_{\text {RES }}\right.$ and FWHM); the second used the same analytes for the calculation of the sensitivity.

\section{Calculation of the SPR curve parameters}

It was considered TOPAS prisms coated with gold $(50 \mathrm{~nm})$ and a hydrophilic solution. The used analytes were: deionized and degassed water and PBS (Phosphate Buffered Saline) and hypochlorite solutions (Figure 3a, b). First, the dry cell image is captured by the optical detector (CCD camera and spectrometer for AIM and WIM, respectively) where only air $(\mathrm{n}=1)$ is present. When water contacts the sensible surface of the prism (wet cell), the surface plasmons are excited and a minimum is observed in the normalized reflectivity. Changes in the analyte (wet cell condition) are repeated along the test cycle and serve to analyze the values of the resonance parameters $\left(\lambda_{\text {RES }}\right.$ and $\left.\theta_{\text {RES }}\right)$ and the width (FWHM). According Figure $3 \mathrm{a}$, a good reproducibility has been obtained considering various test cycles for WIM. When water is changed by hypochlorite solution,
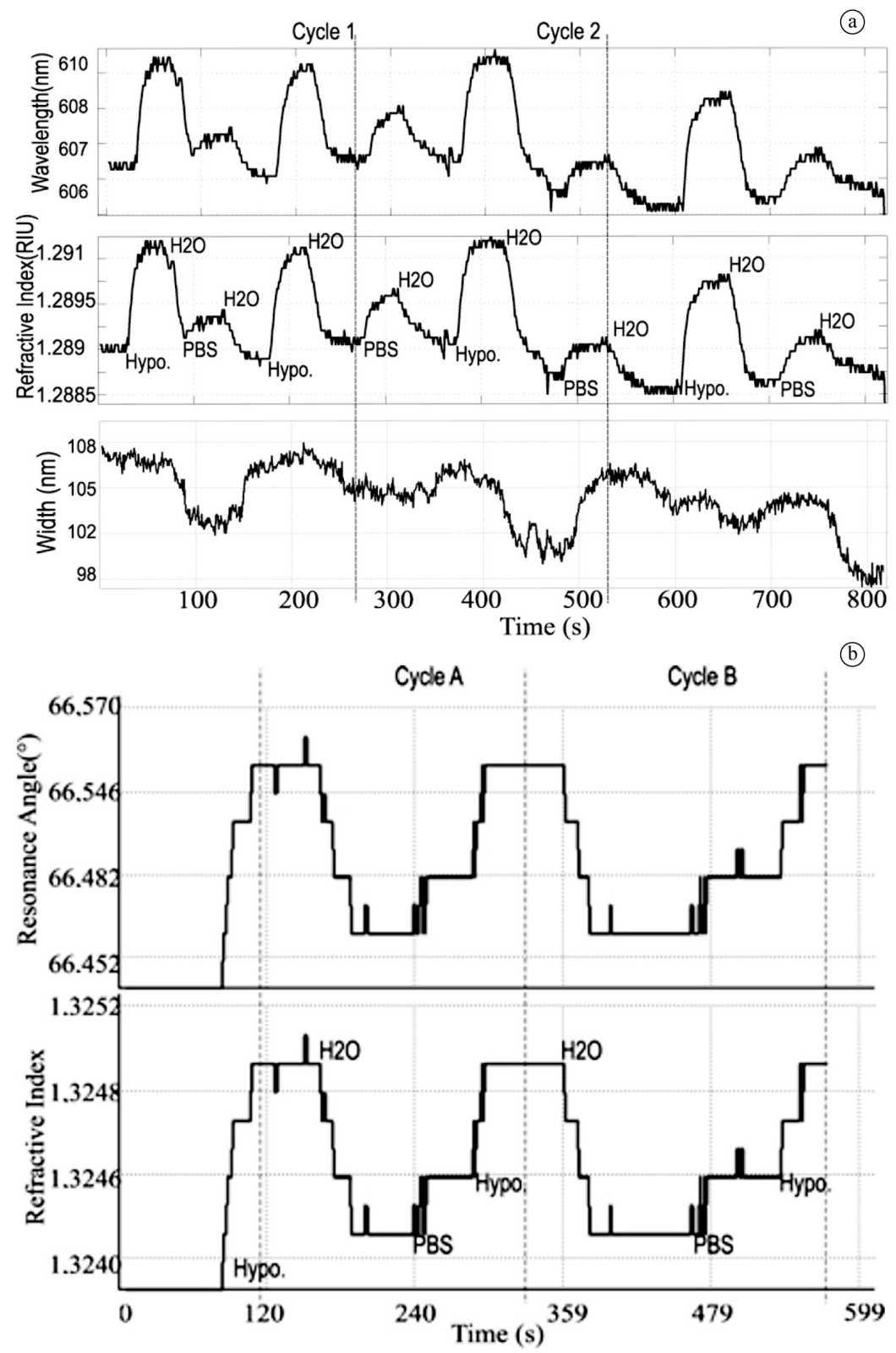

Figure 3. Experimental results for changes in analytes for (a) WIM and (b) AIM cases. 
changes in some parameters have been measured: for refractive index $2.5 \times 10^{-3}$ RIU, which leads to variations on the resonant wavelength $\left(\Delta \lambda_{\mathrm{RES}}=4 \mathrm{~nm}\right)$ and FHWM $(\triangle \mathrm{FWHM}=8 \mathrm{~nm})$.

For AIM case, the transition water-hypochlorite implies the following variations (Figure $3 b$ ): $\Delta \mathrm{n}_{3}=1.1 \times 10^{-3} \mathrm{RIU} ; \Delta \theta_{\mathrm{RES}}=0.07^{\circ}$ and $\Delta \mathrm{FWHM}=3^{\circ}$. Hydrophobic analytes have also used for PPBIO. Figure 4 depicts the sensorgrams only at WIM case considering the circulation of Neutravidin $(0.01 \mathrm{mg} / \mathrm{ml})$ and results when refractive index (a), resonant wavelength (b) and SPR curve width (c) are considered as outputs. Refractive index of analyte $\left(\mathrm{n}_{3}\right)$ variation, after four minutes, was considered over saturation (monolayer condition) with a value of $4 \times 10^{-3} \mathrm{RIU}$ for an associated variation $\Delta \lambda_{\mathrm{RES}}=10.8 \mathrm{~nm}$.

\section{Calculation of sensitivity}

For this experimental test, three different PBS solutions $(50 \%, 77 \%$ e $100 \%)$ have been used with degassed and deionized water. The following procedures have been used for both operation modes:

- Device calibration, based on the air circulation, i.e., dry cell condition;

- Water circulating by the sensing area (wet cell condition);

- Monitoring of the reflectivity and calculation of the SPR response;

- Visualization of sensorgrams;

- Sensitivity calculation for both operation modes.

For AIM case, the experimental cycle was $\mathrm{H}_{2} \mathrm{O} \rightarrow$ $\mathrm{PBS} 50 \% \rightarrow \mathrm{H}_{2} \mathrm{O} \rightarrow \mathrm{PBS} 75 \backslash \% \rightarrow \mathrm{H}_{2} \mathrm{O} \rightarrow \mathrm{PBS} 100 \%$ $\rightarrow \mathrm{H}_{2} \mathrm{O}$. The ambient temperature was $22^{\circ} \mathrm{C}$, the laser temperature was adjusted to $22{ }^{\circ} \mathrm{C}$ and the adjusted current was $24 \mathrm{~mA}$. Then, a hypochlorite solution was used to wash the sensing area in order to obtain the operation point. The obtained results shown a sensitivity of $\mathrm{S}_{\text {AIM }}=126^{\circ} /$ RIU (Figure 5a).

For the WIM case, the used sequence was $\mathrm{H} 2 \mathrm{O} \rightarrow$ $\mathrm{PBS} 100 \% \rightarrow \mathrm{H} 2 \mathrm{O} \rightarrow \mathrm{PBS} 75 \% \rightarrow \mathrm{H} 2 \mathrm{O} \rightarrow \mathrm{PBS}$ $50 \% \rightarrow \mathrm{H} 2 \mathrm{O}$. The same washing process have been used, but here the adequation condition is a resonant wavelength of $670 \mathrm{~nm}$ at the wet cell condition. The obtained sensitivity was $\mathrm{S}_{\mathrm{WIM}}=2261 \mathrm{~nm} / \mathrm{RIU}$ (Figure 5b).

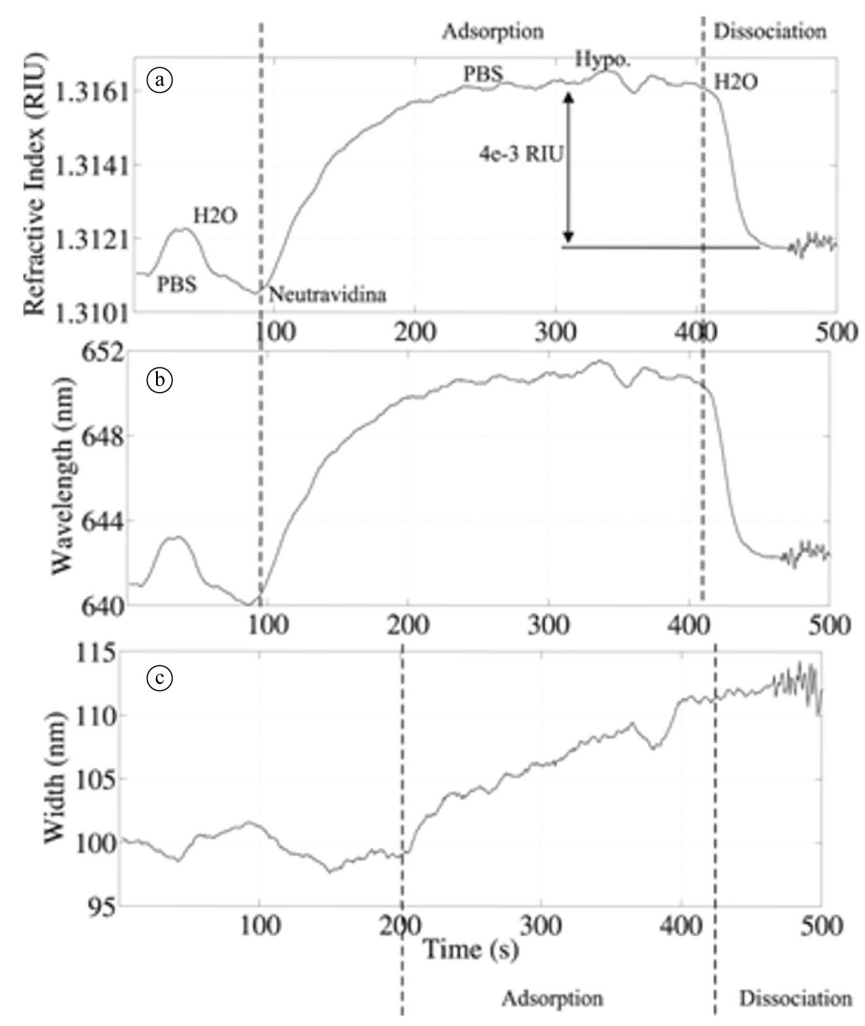

Figure 4. Results for hydrophobic solution (Neutravidin) at WIM case, considering (a) refractive index, (b) resonant wavelength and (c) SPR curve width as outputs. 


\section{Effect of different bimetallic layer combinations}

Combinations of different metallic layers have been used to enhance the sensitivity of SPR based biosensors. Such combinations aim at reducing the final cost of the sensor and also increase the number of possible operating points (OP), i.e., the pair for both the AIM and WIM modes (Oliveira et al., 2013; Oliveira et al., 2014b). Recently, palladium and other metals have been used to provide good characteristics for biological and industrial applications (Zuppella et al., 2015).

Here we discuss the use of thin metallic layer composed of an alloy of two different metals. For this study the dielectric constant will be represented as convex function expressed in terms of its respective volume fractions, i.e., the quantities of these materials at the bimetallic combination. The bimetallic alloy is represented as the layer 2 in the multilayer depicted in Figure 1. The Gold has been chosen for the top layer due to its chemical stability. The possible metal alloys were: $\mathrm{Au} / \mathrm{Al}$ with the following thickness combinations: 20/5nm, 15/10nm, 10/15nm and 5/20nm; Au/Ag and $\mathrm{Au}-\mathrm{Cu}$ with the following thickness combinations: 40/10nm, 30/20nm, 20/30nm and 10/40nm.

Figure 5 shows the sensibility of the bi-metallic layer compared to the gold monolayer sensitivity, i.e, $S_{\lambda_{R}}^{n_{3}}=3435 \mathrm{~nm} / \mathrm{RIU} \$$. Increasing the volume fraction of $\mathrm{Al}$, reduces the sensitivity, especially in WIM, when $80 \%$ of $\mathrm{Al}$ are used: the sensitivity reduces to $92 \%$. Increasing the silver and copper volume fractions could be improved in $20 \%$ with silver and worsened in $16 \%$ with copper. For the AIM gold sensitivity is equal to $123.8^{\circ} / \mathrm{RIU}$. Increasing the volume fraction of $\mathrm{Al}$ and $\mathrm{Ag}$ reduces the sensitivity. Increasing the copper volume fraction essentially don't affect the sensitivity.

\section{Discussion}

Here, a polymer-based surface plasmon resonance biochip has been presented. SPR phenomenon theory,
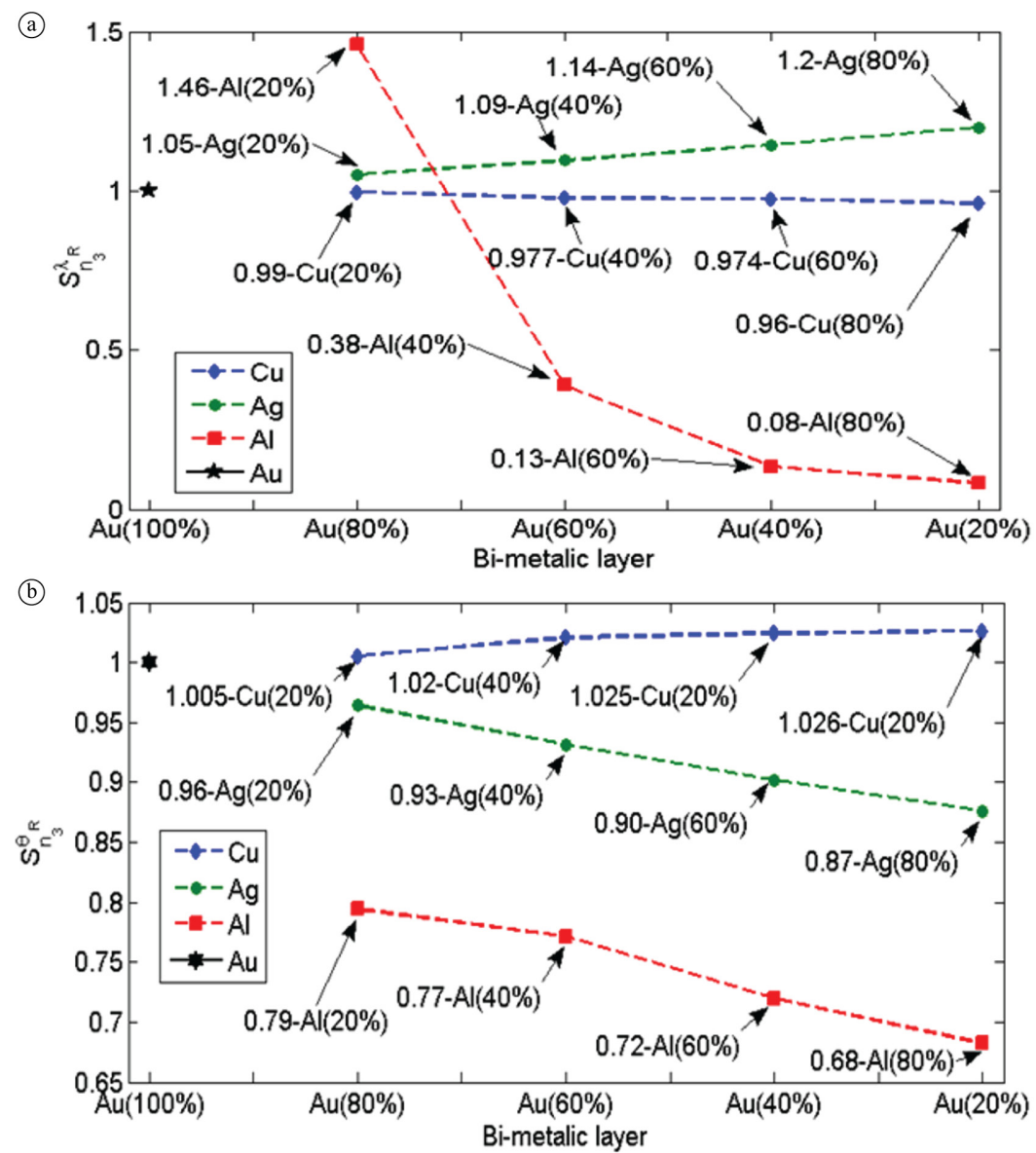

Figure 5. (a) WIM and (b) AIM sensitivities, normalized by gold sensitivity value, for different bi-metallic layer OP with 670 nm and $68^{\circ}$. Black star refers the gold monolayer with $50 \mathrm{~nm}$. 
fundamentals of SPR and LPSR biosensors and the sensing methodologies have also been shown. Construction and experimental aspects of PPBIO biochip was presented in order to elucidate the design, fabrication and use of polymer-based biosensors as alternatives to the conventional ones, which uses glass devices as the optical substrate material. The design and construction aspects, where disposable and low-cost devices has been developed, but requiring a post-processing cycle due to some technical problems from injection molding process, like mechanical stress and birefringence. It is true for majority of the polymer-based SPR biosensors, where the substrate structure is a prism, and normally annealing process is required and then adds more time and associated design cost for the design process. Some future possibilities are the use of silicon-based biochips, where the multilayer receipt for biosensing is deposited over a silicon (or similar material) wafer. It provides, besides a good alternative to solve problems with polymer and glass-based devices, a great possibility to miniaturize the SPR biosensor (DiPippo et al., 2010; Vivien and Pavesi, 2013). Another possibility is the use of microstructured optical fibers as biochips, but they have the complex construction way as a limiting factor (Hassani and Skorobogatiy, 2006; Lee et al., 2011; Yu et al., 2010).

Experimental details have been presented for the PPBIO biochip, where considerations for determination of the resonance parameters and calculation of the sensitivity for hydrophilic and hydrophobic solutions were also presented. Another possibility is the use of bimetallic layers to increase the sensitivity and then provide more adequate devices, as it has been also demonstrated here.

These SPR biosensors are promising analytical devices for point in care and system-on-chip applications, due to advantages like low-cost implementation, possibility to miniaturization and integration with all biosensor elements and portability due to low size and weight. Albeit this, polymer-based SPR biosensors using prisms as the substrates present drawbacks specially related to the mold design, where its design and fabrication are high-cost and complex. Two alternatives are: the optical fibers, that possess low-cost, ease to miniaturization, but they have a complex fabrication; and the nanodevices, which use other physical phenomenon called LPSR and it have been a tendency in the recent years, but it requires more development and research.

\section{Acknowledgements}

The authors would like to thank CNPq and CAPES for the financial support and research grants.

\section{References}

Arima Y, Toda M, Iwata H. Surface plasmon resonance in monitoring of complement activation on biomaterials. Advanced Drug Delivery Reviews. 2011; 63(12):988-99. http://dx.doi.org/10.1016/j.addr.2011.06.018. PMid:21803085.

Atar N, Eren T, Yola ML, Wang S. A sensitive molecular imprinted surface plasmon resonance nanosensor for selective determination of trace triclosan in wastewater. Sensors and Actuators. B, Chemical. 2015; 216:638-44. http://dx.doi. org/10.1016/j.snb.2015.04.076.

Carvalho RM, Rath S, Kubota LT. SPR - Uma nova ferramenta para biosensores. Quimica Nova. 2006; 26:299-304.

Cennamo N, Varriale A, Pennacchio A, Staiano M, Massarotti D, Zeni L, D'Auria S. An innovative plastic optical fiberbased biosensor for new bio/applications. The case of celiac disease. Sensors and Actuators. B, Chemical. 2013; 176:1008-14. http://dx.doi.org/10.1016/j.snb.2012.10.055.

Cennamo N, Zeni L. Bio and chemical sensors based on surface plasmon resonance in a plastic optical fiber. In: Sulaiman Y, Harun SW and Arof H. Optical sensors - new developments and practical applications. Rijeka: INTECH; 2014. p. 119-40.

Chinowsky TM, Quinn JG, Bartholomew DU, Kaiser R, Elkind JL. Performance of the Spreeta 2000 integrated surface plasmon resonance affinity sensor. Sensors and Actuators. B, Chemical. 2003; 91(1-3):266-74. http://dx.doi. org/10.1016/S0925-4005(03)00113-8.

Chinowsky TM, Soelberg SD, Baker P, Swanson NR, Kauffman P, Mactutis A, Grow MS, Atmar R, Yee SS, Furlong CE. Portable 24-analyte surface plasmon resonance instruments for rapid, versatile biodetection. Biosensors \& Bioelectronics. 2007; 22(9-10):2268-75. http://dx.doi. org/10.1016/j.bios.2006.11.026. PMid:17223032.

Dash JN, Jha R. SPR biosensor based on polymer PCF coated with conducting metal oxide. IEEE Photonics Technology Letters. 2014; 26(6):595-8. http://dx.doi.org/10.1109/ LPT.2014.2301153.

DiPippo W, Lee BJ, Park K. Design analysis of doped-silicon surface plasmon resonance immunosensors in mid-infrared range. Optics Express. 2010; 18(18):19396-406. http://dx.doi. org/10.1364/OE.18.019396. PMid:20940835.

Elkind JL, Stimpson DI, Strong AA, Bartholomew DU, Melendez JL. A commercial solution for surface plasmon sensing. Sensors and Actuators. B, Chemical. 1999; 54(12):182-90. http://dx.doi.org/10.1016/S0925-4005(98)00336-0.

Ghosh S, Ray M. Analysis of silicon based surface plasmon resonance sensors with different amino acids. Silicon. 2015; 7(4):313-22. http://dx.doi.org/10.1007/s12633-015-9293-8.

Guo J, Keathley PD, Hastings JT. Dual-mode surfaceplasmon-resonance sensors using angular interrogation. Optics Letters. 2008; 33(5):512-4. http://dx.doi.org/10.1364/ OL.33.000512. PMid:18311309.

Hassani A, Skorobogatiy M. Design of the microstructured optical fiber-based surface plasmon resonance sensors with enhanced microfluidics. Optics Express. 2006; 14(24):1161621. http://dx.doi.org/10.1364/OE.14.011616. PMid:19529581. 
Hoa XD, Kirk AG, Tabrizian M. Towards integrated and sensitive surface plasmon resonance biosensors: A review of recent progress. Biosensors \& Bioelectronics. 2007; 23(2):151-60. http://dx.doi.org/10.1016/j.bios.2007.07.001. PMid:17716889.

Homola J, Vaisocherová H, Dostálek J, Piliarik M. Multianalyte surface plasmon resonance biosensing. Methods. 2005; 37(1):26-36. http://dx.doi.org/10.1016/j.ymeth.2005.05.003. PMid:16199172.

Homola J. Surface plasmon resonance based sensors. Amsterdam: Springer; 2006.

Hu C, Gan N, Chen Y, Bi L, Zhang X, Song L. Detection of microcystins in environmental samples using surface plasmon resonance biosensor. Talanta. 2009; 80(1):407-10. http:// dx.doi.org/10.1016/j.talanta.2009.06.044. PMid:19782244.

Kasztelanic R. Parallel multichannel architecture for surface plasmon resonance sensors. Journal of the European Optical Society. 2012; 7:12038. http://dx.doi.org/10.2971/ jeos.2012.12038.

Kuang KSC, Cantwell WJ, Scully PJ. An evaluation of a novel plastic optical fiber sensor for axial strain and bend measurements. Measurement Science \& Technology. 2002; 13(10):1523-34. http://dx.doi.org/10.1088/09570233/13/10/303.

Kumbhat S, Sharma K, Gehlot R, Solanki A, Joshi V. Surface plasmon resonance based immunosensor for serological diagnosis of dengue virus infection. Journal of Pharmaceutical and Biomedical Analysis. 2010; 52(2):255-9. http://dx.doi. org/10.1016/j.jpba.2010.01.001. PMid:20097030.

Le Person JL, Colas F, Compère C, Lehaitre $\mathrm{M}$, Anne M-L, Boussard-Plédel C, Bureau B, Adam J-L, Deputier S, Guilloux-Viry M. Surface plasmon resonance in chalcogenide glass-based optical system. Sensors and Actuators. B, Chemical. 2008; 130(2):771-6. http://dx.doi.org/10.1016/j. snb.2007.10.067.

Lee HW, Schmidt MA, Russell RF, Joly NY, Tyagi HK, Uebel P, Russell P. Pressure-assisted melt-filling and optical characterization of $\mathrm{Au}$ nano-wires in microstructured fibers. Optics Express. 2011; 19(13):12180-9. http://dx.doi. org/10.1364/OE.19.012180. PMid:21716455.

Liedberg B, Nylander C, Lundström I. Surface plasmon resonance for gas detection and biosensing. Sensors and Actuators. 1983; 4: 299-304.

Loureiro FCCL, Barreto AGS No, Lima AMN, Moreira CS, Neff H. Molecular transport and mutual diffusion measurement method in a micro-fluidic system, based on surface plasmon resonance spectroscopy. Procedia Chemistry. 2009; 1(1):1099-102. http://dx.doi.org/10.1016/j. proche.2009.07.274.

Loureiro FCCL, Barreto AGS No, Moreira CS, Lima AMN, Neff $\mathrm{H}$. A method for determining the mutual diffusion coefficient of molecular solutes based on surface plasmon resonance sensing. Sensors and Actuators. B, Chemical. 2011; 154(2):129-36. http://dx.doi.org/10.1016/j.snb.2010.02.023.

Maier SA. Plasmonics: fundamentals and applications. New York: Springer; 2007.

Moreira CS, Barreto AGS No, Lima AMN, Thirstrup C, Neff H. Exchangeable low cost polymer biosensor chip for surface plasmon resonance spectroscopy. Procedia Chemistry. 2009; 1(1):1479-82.

Moreira CS, Lima AMN, Neff H, Thirstrup C. Temperaturedependent sensitivity of surface plasmon resonance sensors at the gold-water interface. Sensors and Actuators. B, Chemical. 2008; 134(2):854-62. http://dx.doi.org/10.1016/j. snb.2008.06.045.

Moreira CS. Projeto e realização de um biochip óptico para aplicações biológicas baseado no principio de ressonância de plásmons de superfície [thesis]. Campina Grande: Universidade Federal de Campina Grande; 2010.

Mukhopadhyay R. Surface plasmon resonance instruments diversify. Analytical Chemistry. 2005; 77(15):313A-7A. http://dx.doi.org/10.1021/ac053440n.

Myszka DG, Rich RL. Implementing surface plasmon resonance biosensors in drug discovery. Pharmaceutical Science \& Technology Today. 2000; 3(9):310-7. http://dx.doi. org/10.1016/S1461-5347(00)00288-1. PMid:10996572.

Neff H, Zong W, Lima AMN, Borre M, Holzhüter G. Optical properties and instrumental performance of thin gold films near the surface plasmon resonance. Thin Solid Films. 2005; 21:1745-52.

Oliveira LC, Moreira CS, Barreto AGS No, Lima AMN, $\mathrm{Neff} \mathrm{H}$. Effect of different bimetallic layer combinations on the sensitivity of a SPR polymer biochip. In: Proceedings of the 15th International Meeting on Chemical Sensors IMCS 2014; 2014 Mar 16-19; Buenos Aires, Argentina. Buenos Aires: IMCS; 2014a. p. 80.

Oliveira LC, Moreira CS, Barreto AGS No, Lima AMN, Neff H. Manufacturing and operational considerations for a polymeric based SPR biochip. In: Proceedings of the 15th International Meeting on Chemical Sensors - IMCS 2014; 2014 Mar 16-19; Buenos Aires, Argentina. Buenos Aires: IMCS; 2014b. p. 98.

Oliveira LC, Moreira CS, Thirstrup C, Melcher EUK, Lima AMN, Neff H. A surface plasmon resonance biochip that operates both in the angular and wavelength interrogation modes. IEEE Transactions on Instrumentation and Measurement. 2013; 62(5):1223-32. http://dx.doi.org/10.1109/ TIM.2012.2232433.

Oliveira LC. Sistema computacional para biossensor baseado na ressonância de plasma de superfície [dissertation]. Campina Grande: Universidade Federal de Campina Grande; 2011.

Otto A. Excitation of nonradiative surface plasma waves in silver by the method of frustrated total reflection. Zeitschrift für Physik A Hadrons and Nuclei. 1968; 216:398-410.

Patskovsky S, Kabashin AV, Meunier M, Luong JHT. Silicon-based surface plasmon resonance sensing with two surface plasmon polariton modes. Applied Optics. 2003; 42(34):6905-9. http://dx.doi.org/10.1364/AO.42.006905. PMid:14661802.

Raether H. Surface plasmons on smooth and rough surfaces and on gratings. Berlin: Springer-Verlag Berlin Heidelberg; 1988. (Springer Tracts in Modern Physics, 111).

Rivero PJ, Urrutia A, Goicoechea J, Arregui FJ. Optical fiber humidity sensors based on localized surface plasmon resonance (LSPR) and lossy-mode resonance (LMR) in 
overlays loaded with silver nanoparticles. Sensors and Actuators. B, Chemical. 2012; 173:244-9. http://dx.doi. org/10.1016/j.snb.2012.07.010.

Rusnati M, Presta M. Angiogenic growth factors interactome and drug discovery: the contribution of surface plasmon resonance. Cytokine \& Growth Factor Reviews. 2015; 26(3):293-310. http://dx.doi.org/10.1016/j.cytogfr.2014.11.007. PMid:25465594.

Santiago MFS, Silva TB, Mozzini MH, Coutinho IBG, Medeiros ES, Cruz RMS, Moreira CS. Construction aspects of a plastic optical fiber-based surface plasmon resonance biochip. Proceedings of the 24th Optical Fiber Conference; 2015 Sept 28 - Oct 02; Curitiba, Brasil. Curitiba: UTFPR; 2015.

Schasfoort RBM, Tudos AJ. Handbook of surface plasmon resonance. Cambridge: RCS Publishing; 2008.

Sener G, Uzun L, Say R, Denizli A. Use of molecular imprinted nanoparticles as biorecognition element on surface plasmon resonance sensor. Sensors and Actuators. B, Chemical. 2011; 160(1):791-9. http://dx.doi.org/10.1016/j. snb.2011.08.064.

Sexton BA, Feltis BN, Davis TJ. Characterization of gold surface plasmon resonance sensor substrates. Sensors and Actuators. A, Physical. 2008; 141(2):471-5. http://dx.doi. org/10.1016/j.sna.2007.10.020.

Sharma AK, Jha R, Gupta BD. Fiber-optic sensors based on surface plasmon resonance: a comprehensive review. IEEE Sensors Journal. 2007; 7(8):1118-29. http://dx.doi. org/10.1109/JSEN.2007.897946.

Šípová H, Homola J. Surface plasmon resonance sensing of nucleic acids: a review. Analytica Chimica Acta. 2013; 773:9-23. http://dx.doi.org/10.1016/j.aca.2012.12.040. PMid:23561902.

Situ C, Mooney MH, Elliott CT, Buijs J. Advances in surface plasmon resonance biosensor technology towards high-throughput, food-safety analysis. Trends in Analytical Chemistry. 2010; 29(11):1305-15. http://dx.doi.org/10.1016/j. trac.2010.09.003.

Souza CA Fo. Desenvolvimento de um Sistema Eletrônico de Aquisição e Processamento para Biosensores [dissertation].
Campina Grande: Universidade Federal de Campina Grande; 2006.

Thillaivinayagalingam P, Gommeaux J, McLoughlin M, Collins D, Newcombe AR. Biopharmaceutical production: applications of surface plasmon resonance biosensors. Journal of Chromatography. B, Analytical Technologies in the Biomedical and Life Sciences. 2010; 878(2):149-53. http:// dx.doi.org/10.1016/j.jchromb.2009.08.040. PMid:19762290

Thirstrup C, Zong W, Borre M, Neff H, Pedersen HC, Holzhueter G. Diffractive optical coupling element for surface plasmon resonance sensors. Sensors and Actuators. B, Chemical. 2004; 100(3):298-308. http://dx.doi.org/10.1016/j. snb.2004.01.010.

Vasile GC, Vasile IM, Sava V. A method to remove optical fibers coating. UPB Science Bulletin. 2013; 75:155-60.

Vivien L, Pavesi L. Handbook of Silicon Photonics. Boca Raton: CRC Press. 2013

Wang T-J, Tu C-W, Liu F-K, Chen H-L. Surface plasmon resonance waveguide biosensor by bipolarization wavelength interrogation. IEEE Photonics Technology Letters. 2004; 16(7):1715-7. http://dx.doi.org/10.1109/LPT.2004.828376.

Willets KA, Van Duyne RP. Localized surface plasmon resonance spectroscopy and sensing. Annual Review of Physical Chemistry. 2007; 58(1):267-97. http://dx.doi.org/10.1146/ annurev.physchem.58.032806.104607. PMid:17067281.

Yu X, Zhang Y, Pan S, Shum P, Yan M, Leviantan Y, Li C. A selectively coated photonic crystal fiber based surface plasmon resonance sensor. Journal of Optics. 2010; 12(1).

Yu R, Shibayama T, Meng X, Takayanagi S, Yoshida Y, Yatsu S, Watanabe S. Effects of nanosecond-pulsed laser irradiation on nanostructure formation on the surface of thin $\mathrm{Au}$ films on $\mathrm{SiO} 2$ glass substrates. Applied Surface Science. 2014; 289:274-80. http://dx.doi.org/10.1016/j.apsusc.2013.10.149.

Zuppella P, Pasqualotto E, Zuccon S, Gerlin F, Corso AJ, Scaramuzza M, De Toni A, Paccagnella A, Pelizzo MG. Palladium on plastic substrates for plasmonic devices. Sensors. 2015; 15(1):1138-47. http://dx.doi.org/10.3390/ s150101138. PMid:25585102.

\section{Authors}

\footnotetext{
Cleumar da Silva Moreira ${ }^{1 *}$, Leiva Casemiro Oliveira ${ }^{2}$, Robert Fischer ${ }^{3}$, Eliton Souto Medeiros ${ }^{4}$, Antonio Marcus Nogueira Lima ${ }^{2}$, Helmut Neff ${ }^{2}$

${ }^{1}$ Electrical Engineering Department, Instituto Federal da Paraíba - IFPB, Av. João da Mata, 256, Jaguaribe, CEP 58015-020, João Pessoa, PB, Brazil.

${ }^{2}$ Biosensors Laboratory, Universidade Federal de Campina Grande - UFCG, Campina Grande, PB, Brazil.

${ }^{3}$ Physics Department, Universidade Federal do Rio Grande do Sul - UFRGS, Porto Alegre, RS, Brazil.

${ }^{4}$ Materials Engineering Department, Universidade Federal da Paraíba - UFPB, João Pessoa, PB, Brazil.
} 